\title{
Anesthesiology, Autonomy, and Justice: Getting It Right
}

\author{
T Papadimos, A Marco, G Filatoff
}

\section{Citation}

T Papadimos, A Marco, G Filatoff. Anesthesiology, Autonomy, and Justice: Getting It Right. The Internet Journal of Anesthesiology. 2004 Volume 9 Number 1.

\begin{abstract}
Ethics in medicine is of great importance. The Accreditation Council for Graduate Medical Education (ACGME) has determined that Professionalism (including ethics) should be a core competency requirement in resident education. Anesthesiologists will be involved in situations where sound knowledge of ethics principles will be of value in guiding appropriate patient care and coordination of that care. In this report we highlight the importance of patient autonomy and justice, the need for understanding ethics principles, and the value of the formal education of our residents in ethics.
\end{abstract}

\section{INTRODUCTION}

Ethics in medicine has come to the socio-political foreground ${ }_{{ }_{1}, 2,3,4}$ The Accreditation Council for Graduate Medical Education (ACGME) has indicated to residency training programs that Professionalism (including ethics) is a core competency that is a required component of education ${ }_{5}$. Anesthesiologists may be involved in situations where sound knowledge of ethics principles will guide appropriate care and coordination of care for the patient.

We present a case where the ethical principles of autonomy and justice in regard to a patient whose capacity to make a decision was questioned are highlighted. Awareness and education in medical ethics are of paramount importance to those practicing anesthesiology. Anesthesiologists should not be bystanders when cases that raise ethical questions/issues are brought to the operating suites. When a clinician requests anesthesiology services, he or she is asking for a consult. An anesthesiologist may need to step forward and interject his or her expertise as a consultant in regard to the ethics of a clinical situation with which they will be involved.

\section{CASE REPORT}

A 45 year old female presented for a repeat aortic valve replacement (AVR), her fourth sternotomy. She had a history of bipolar disorder and an inability to recall recent events more than two hours old. She had been institutionalized in a group home for many years with good functioning. Additional past medical history was significant for coronary artery disease, hypertension, hyperlipidemia, 12 pack-years of tobacco use, and 2 ounces of alcohol per week.
Her surgical history included repair of an atrial-septal defect at age 3, coronary artery bypass grafting (CABG) in 1992, an AVR-CABG in 1994 (St. Jude's valve), cholecystectomy, hysterectomy, and a craniotomy in 2002 for a spontaneous hematoma. Her medications consisted of docusate sodium, iron, aspirin, aripiprazole, escitalopram, venlafaxine, lamotrigine, furosemide, potassium, olanzapine, metoprolol, pantoprazole, acetaminophen and oxycodone, atorvastatin, estrogen, and warfarin.

She refused and was unable to follow the anticoagulation regimen presented by her physicians, even though her caretakers endeavored to gain her compliance. A recent transesophageal echocardiogram (TEE) indicated that a thrombus had formed on the mechanical aortic valve. Other TEE findings were mild mitral regurgitation, mild tricuspid regurgitation, and mild aortic regurgitation, the left ventricular ejection fraction was greater than $50 \%$, and the E:A ratio was normal.

Discussions among psychiatry, cardiology, and cardiothoracic surgery consultants concluded that the patient would be best served by prophylactic surgical intervention in which the patient's mechanical valve would be replaced with a tissue valve, thus eliminating the need for anticoagulation. The medical and surgical consultants acknowledged that there were non-surgical alternatives to this treatment, but that the patient's lack of cooperation would result in further complications.

In the preoperative holding area, the patient's inability to recall conversations more that two hours old concerned the anesthesiology team. Her medical chart indicated the 
necessity for multiple reiterations of her condition, the medical and surgical plan, and her options. The anesthesiology team delayed the case for one hour and instigated a serious discussion of autonomy and justice between the patient, the anesthesiology team, and the other medical consultants. Ultimately, the surgery was undertaken, and the mechanical aortic valve was successfully replaced with a tissue valve. The patient was discharged without incident to a rehabilitative facility 10 days after surgery.

\section{DISCUSSION}

The Department of Anesthesiology was consulted before the surgery, but the resident performing the preoperative evaluation did not recognize the ethics issues and, thus, did not follow through with further evaluation/discussions. The presentation of a patient with such a history should be of concern to the responsible attending anesthesiology consultant. While as clinicians we usually focus on the hemodynamics and physiology, the real issue here is ethics, not how we do the case, but why we should do it. Issues of autonomy and justice push the practice of anesthesiology beyond the confines of pharmacology, physiology, and anatomy. This case illustrates why the ACGME has recommended the addition of Professionalism (including ethics) to the residency curriculum as one of the six core competencies. A member of the anesthesiology team was coincidentally a member of the hospital ethics committee and was familiar with such issues and their principles. The case was delayed to allow the anesthesiology team time to address their concerns.

Our first concern, as ethicists, was the issue of autonomy. Respect for autonomy is a deeply rooted moral principle in Western societies. Autonomy refers to self-will or governance and has two conditions that are absolutely essential: (1) liberty (freedom from controlling influences) and (2) agency (capacity for intentional action).

Consequential decisions such as surgery cannot be fully autonomous but should be substantially autonomous. We would expect a patient who is autonomous to choose a course of action (1) intentionally, (2) with an ability to understand, and (3) without a controlling influence that could determine such an action. 6

In this case the patient understood her situation for the period of time her recent memory remained intact (1-2 hours) and during this time it was evident her intent was to follow a course of action that she felt to be in her selfinterest (to have the surgery). The issue of controlling influences needed to be addressed because it was clear that taking an oral medication was a simpler proposition. Was she trying to please the caregivers in her residential situation? Were the caregivers or her family pressuring her to have the procedure so as to alleviate the requirements of vigilant care on their part? Did she have the capacity to recognize the presence of controlling influences during her periods of functional memory?

Beauchamp and Childress distinguish judgments of capacity from judgments of competence on the grounds that "health professionals assess capacity and incapacity whereas courts determine competence and incompetence". ${ }_{6}$ Only courts can establish legal incompetence. Physicians cannot declare patients incompetent as a matter of law, but they can override or constrain patient decision-making in certain circumstances. ${ }_{6}$ In this case the general consensus was that the capacity of the patient was intact.

The elements of informed consent are (1) competence, (2) disclosure, (3) understanding, (4) voluntariness, and (5) consent. ${ }_{6}$ In the time period of the patient's recent memory capabilities (1-2 hours before surgery) these five elements were addressed. Anesthesiologists play an important role on the health care team and should take every opportunity to be advocates for patients and society in general. According to Immanuel Kant, "Freedom is the power to will an end to an action for myself" ${ }_{7}$. An action that originates with the patient can only be attributed to the patient, and is therefore truly the patient's decision. The patient reflected upon her decision during periods of recollection (even though decisions, options, and events had to be re-explained on various occasions). In choosing surgery she was participating in a free action: one that is brought into being by reason alone. ${ }_{7}$ The decision to go forward with surgery arose out of a rational process, albeit one with confined temporal limits.

The concept of justice goes back thousands of years. The ancient Greek Stoics claimed that justice was a virtue that arose from social instincts and forbade one man to injure another, to take his property, or to take property that belonged to the community. Additionally, justice to the Stoics was an active beneficence that formed societal bonds. ${ }_{8}$ John Locke set out the claim to rights of life, liberty, and property as Americans now understand them ${ }_{9}$ and David Hume reminds us that, "History, experience, and reason sufficiently instruct us in this natural progress of human sentiments, and in the gradual enlargement of our regards to 
justice, in proportion as we become acquainted with extensive utility of that virtue". ${ }_{10}$ Today's view of justice is criminal (infliction of punishment), rectificatory (compensation for malpractice and breaches of contracts), and distributive (fair, equitable, and appropriate distribution). ${ }_{11}$

The anesthesiology team's concern was that the patient receive the correct and proper health care and to ensure that the patient understood she not only had the right to health care, but that she was aware of the various available options. Although the question of autonomy was settled, the issue of justice was still present. The patient had the right to refuse anticoagulation and she had the right to have the proposed procedure. However, the question of whether this surgery was a fair and equitable alternative to perpetrate upon one's self needed to be addressed. Furthermore, the fairness to society to incur the cost of the surgery is another legitimate interrogative, but it cannot be addressed at a patient's bedside and is beyond the scope of this report. Since justice (distributive) to the patient was not compromised (as opposed to the possible inequity to society in general), with the issues concerning autonomy settled, and in light of the fact that medical consultants felt there was not a better alternative than replacement of the mechanical aortic valve with a tissue valve, the surgery proceeded.

Training in ethics, along with the anesthesiologist's role as a perioperative physician, assists a practitioner in the determination of the appropriate contribution to the patient's welfare (principle of beneficence) and appropriate examination of the situation so as to not inflict any harm (principle of nonmaleficence). As to the principle of beneficence, it is defined as an obligation to "help others further their important and legitimate interests". ${ }_{12}$ In this case the patient's legitimate interest was getting her surgery performed, that is, if it was the best alternative as a plan of care. Another legitimate interest was ensuring she understood, as best as possible, what the surgical procedure involved. Nonmaleficence is also very important in this situation. Anesthesiologists should use their skills to help the sick according the their abilities and judgments, but in so doing, not to wrong or injure the patient. Allowing the patient to submit to a procedure that he or she does not understand or does not need is a "wrong" that could lead to mental or physical injury. 13

The vigilant anesthesiologist will have to add the principles of medical ethics to his or her armamentarium. Under no circumstances should an anesthetic be performed without the issue of autonomy being settled. Ethics issues should be addressed and resolved preoperatively. Any time there is a lack of patient autonomy an opportunity for the violation of the three remaining principles of biomedical ethics, justice, beneficence, and nonmaleficence, presents itself.

Each case is different. Just as one anesthetic does not fit all, the bioethics of each case may vary. Hospital ethics committees or staff ethicists are available at most hospitals to assist the medical staff. In this case the hospital ethics committee was not involved, but all of the parties involved (physicians and family members) worked together for the benefit of the patient. Anesthesiologists play a vital preoperative role in patient preparation and safety and should not be bystanders.

Formal education for residents in ethics is vital. The ACGME Outcome Project involving Professionalism (including ethics) as it relates to residency training is an important component of the well-rounded and prepared practitioner. Departments of Anesthesiology should endeavor to instill the importance of ethics in their residents. Problem-based learning sessions and bedside consultations with attending physicians offer opportunities to enlighten trainees as to the advantages of incorporating ethics considerations into the everyday practice of medicine.

Anesthesiologists should be willing participants regarding patient and institutional bioethics and not allow their exclusion from a decision-making process in which they will be asked to provide crucial and substantial care to patients with such issues. The anesthesiology community's involvement in issues of ethics as teachers, practitioners, and members of society at large, truly, is of paramount importance.

\section{CORRESPONDENCE TO}

Thomas J. Papadimos, M.D., M.P.H. Department of Anesthesiology Medical College of Ohio 3000 Arlington Avenue Toledo, Ohio 43614, USA Phone: 419-383-3556

Fax: 419-383-3550 Email: tpapadimos@mco.edu

\section{References}

1. Turner L. Bioethics in pluralistic societies. Med Health Care Philos 2004;7:201-28

2. Rubin SB, Zoloth L. Clinical ethics and the road less taken: mapping the future by tracking the past. J Law Med Ethics 2004;32:218-25.

3. Papadimos TJ, Marco AP. The obligation of physicians to medical outliers: A Kantian and Hegelian Synthesis. BMC Med Ethics 2004;5:E3. 
4. Papadimos TJ, Papadimos AT. The student and the ovum: The lack of autonomy and informed consent in trading genes for tuition. Reprod Biol Endocrinol 2004;2:56.

5. Accreditation Council for Graduate Medical Education. Outcome project. Available at:

http::/www.acgme.org/outcome/project. Accessed

November 5, 2004.

6. Beauchamp TL, Childress JF: Respect for Autonomy, Principles of Biomedical Ethics, 5th edition. New York, Oxford University Press, 2001, pp 57-69.

7. Scruton R. Kant: A very short introduction, 1st edition (revised). New York, Oxford University Press, 2001, pp 79-81.

8. Sanbach FH: The Stoics, 2nd edition. Indianapolis, Hacker Publishing, 1994, pp 28-68.
9. Almond B: Rights, A Companion to Ethics, 1st edition. Edited by Singer P. New York, Oxford University Press, 1993, pp 259-69.

10. Hume D: An Enquiry Concerning the Principles of Morals, 2nd edition. La Salle, Open Court Publishing, 1966, pp 25.

11. Beauchamp TL, Childress JF: Justice, Principles of Biomedical Ethics, 5th edition. New York, Oxford University Press, 2001, pp 226-30.

12. Beauchamp TL, Childress JF: Beneficence, Principles of Biomedical Ethics, 5th edition. New York, Oxford University Press, 2001, pp 65-224.

13. Beauchamp TL, Childress JF: Nonmaleficence, Principle of Biomedical Ethics, 5th edition. New York, Oxford University Press, 2001, pp 113-164. 


\section{Author Information}

Thomas J. Papadimos, M.D., M.P.H.

Assistant Professor, Department of Anesthesiology, Medical College of Ohio

Alan P. Marco, M.D., M.M.M.

Associate Professor, Department of Anesthesiology, Medical College of Ohio

Gregory A. Filatoff, M.D.

CA-3 Resident, Department of Anesthesiology, Medical College of Ohio 